\title{
Ensemble interpolation methods for spatio-temporal data modelling
}

\author{
Philip Sallis \\ Geoinformatics Research Centre \\ Auckland University of Technology \\ Auckland, New Zealand \\ psallis@aut.ac.nz
}

\author{
Sergio Hernandez \\ Laboratorio de procesamiento \\ de información geoespacial \\ Universidad Catolica del Maule \\ Talca, Chile \\ shernandez@ucm.cl
}

Abstract- Real time weather forecasting is a highly influential tool in decision making for agriculture. Geographic Information Systems (GIS) can be built to provide information about topographic data such as elevation and distance to oceans or water reservoirs. This data has begun to have increased availability, providing easier access for developing new applications. By using geographic information together with terrestrial measurements from weather stations, the spatial and temporal scales of the climatic variables can be analyzed by interpolation and forecasting.

Most of the interpolation methods provided in common GIS tools are only related to the spatial domain, limiting its use in numerical modelling and prediction of climatic states. However, by adopting a Bayesian approach, it appears possible to estimate the dynamic behaviour of the unobserved climate pattern using a state-space representation. Using this framework, the ensemble Kalman filter or a more general sequential Monte Carlo method could be used for the estimation procedure. A wireless sensor network providing continuous data to populate such a model is described here for potential application of this approach.

Keywords-climatemodelling;interpolation;ensemble methods; kalman filters; GIS, Wireless sensor Networks

\section{INTRODUCTION}

Meteorological models for weather forecasting have been proved to be useful for strategic planning and management in agriculture $[1,2]$ even though some precision is mitigated by random events in nature [3].

The success of agro-climatic models is dependent on the range and resolution of the forecast [4] so adequate physical modelling is required for determining the state of the atmospheric values. In the early 1960's, researchers and engineers from different fields were concerned with the problem of forecasting non-stationary dynamic signals. The focus in meteorology using data assimilation was mainly based on deterministic non-linear filters, where the dynamic model is a perfect representation of the physical system [5]

Nevertheless, one of the main issues in weather forecasting systems based on data assimilation is their sensitivity to initial conditions, which puts fundamental limits on the prediction.

In general terms interpolation methods for geospatial data modelling are best appreciated when we consider how changes occur from one data point to another over time. Change point analysis [6] improves the detection of variable value shifts, especially over large historical sets of data. We consider this precision to be essential for micro-climate modelling where time intervals are typically small because of the short topographic distances between data points and yet condition changes can be large. For this reason, when we model a large historical climate data set (described below) we ask the questions, did a change occur? Did more than one change occur? When did the changes occur? With what confidence can we state that the changes did occur? We have adopted this analytical 
framework approach because change point analysis is a method capable of detecting multiple changes and for climate variation plotting we need to incorporate multiple levels of abstraction from the data we are observing.

\section{INTERPOLATION METHODS}

Linear least squares estimation algorithms in the form of the GeoStatistical Kriging methods [7] are probably the most popular for geospatial interpolation because they enable the prediction of unknown data point conditions (values) determined from a known set of values from neighboring data points. This way we can model change across a plane with a high degree of value expectation certainty. Data points with georeferenced values for latitude and longitude ( $\mathrm{x}$ and y) can be interpolated with their elevation data $(z)$ to provide a terrain map in three dimensions. The greater the number of data points the better the expectation confidence. So when values from three or more data points are known, greater precision can be observed in the output value.

When we describe Ensemble methods [8] for modelling geospatial data, we do so holistically, which is an intrinsic attribute of the approach. These methods provide even greater estimation precision to the data model because they utilise a multiple analysis approach and apply several hypothesis algorithms to a single learning proposition. A refinement of the hypotheses is possible when taking this approach because the intrinsic learning algorithm of the method prunes so-called weak learners to focus on the strength of results produced by one of them. This method is more computationally intensive than using for example, a single supervised neural network algorithm.

While modelling of historical climate data is useful for anticipating future trends, there does exist a challenge for modelling continuous rather than discrete point data. This challenge forms part of our ongoing work. Synoptic and planetary circulation models [9] provide a large scale hydrodynamic approximation to the climatic patterns while mesoscale circulation models are used to characterize horizontal scale, which are smaller than the synoptic scale. Because of the lack of a high resolution meteorological network, mesoscale models such as MM5 and MOS [10] have been used for forecasting surface meteorological and agroclimatic variables in central Chile. The authors of this research reported a spatio-temporal interpolation method for temperature, wind speed, relative humidity, and daily solar radiation in grid cells with a spatial resolution of 15 kms.

In another context, the signal processing community has been interested in stochastic linear filters for signal tracking with uncertain observations. In this case, the dynamic model is not perfect and it is considered as being corrupted with random noise. The Ensemble Kalman filter $($ EnKF)[11] has been proposed in data assimilation situations to model uncertain initial conditions in numerical weather prediction. The EnKF overrides the linearity assumption of the standard Kalman filter by using a Monte Carlo approximation of the optimal probability forecast. Because of the inherent so-called 'curse of dimensionality problem' of stochastic approximation methods such as with a sequential Monte Carlo, the EnKF uses a low-rank approximation to the covariance of the posterior density, which also introduces spurious correlations in the filter estimates.

\section{APPLICATION OF THE ENSEMBLE METHOD}

Experiments with forecasting climatic states using spatial interpolation and ensemble methods is ongoing in the research programme to which this paper refers. Because it is an early stage project no results are yet available but we offer our approach as being conceptually appropriate for the problem domain. Only now is the wireless sensor network (WSN) intended for model propagation being installed and a large set of data will eventually be available for analysis and modeling. We intend to use a Digital Elevation Model (DEM) and the distance from the sea generated by a Geographic Information System 
(GIS) to interpolate temperature from the weather stations [12]. The interpolated values will then be used as observations for a sequential Monte Carlo method for estimating the dynamic climate pattern.

It should be noted here that obtaining a large set of complete data including elevations is not as simple as it might seem. In our ongoing work we are assembling similar data sets from both Chile, see [13] for an example of integrating macro and micro climate data for frost prediction in Chilean vineyards and New Zealand [14] for comparative purpose to test the interpolation method and observe the output models for similarities and differences. A wider research programme to which the work described in this paper relates, consists of a wireless sensor network (WSN) with 28 data collection stations in 8 countries. Each station has a minimum of three sensor arrays, each consisting of 17 sensors for atmosphere, climate, plant and soil. This research relates to monitoring and modeling in viticulture, horticulture and agriculture for precision information provision with a view to effective sustainable management [15]. The accession of this data is expected to provide a large volume of continuous data suitable for our ongoing work with change point analysis and ensemble methods.

\section{SPATIAL INTERPOLATION OF CLIMATIC VARIABLES}

State-space modelling for land surface temperature forecasting [16] is an integral component of our approach. A state-space model contains two equations for describing the dynamic behaviour of the system and the observational process. As seen below the state-space representation is conceptually a graph for sequential probabilistic inference over a partially observed stochastic process. The state $\mathrm{x}$ is an unobserved first-order Markov process and the observations are conditionally independent given the state process.

$x_{k}=f\left(x_{k-1}, v_{k}\right)$ process equation

$z_{k}=g\left(x_{k}, w_{k}\right) \quad$ observation equation
The state of the system $x_{k}$ at time $\mathrm{k}$ is a Markov process observed via the measurement $\mathrm{Z}_{\mathrm{k}}$. The noise sources $\mathrm{v}_{\mathrm{k}}$ and $\mathrm{w}_{\mathrm{k}}$ are assumed as being mutually independent and identically-distributed (i.i.d.) sequences of random variables, which are also independent of the state and the observations $\mathrm{x}_{\mathrm{k}}$ and $\mathrm{z}_{\mathrm{k}}$ respectively. The functions $\mathrm{f}$ and $\mathrm{g}$ represent possibly non-linear mappings from $\mathrm{x}_{\mathrm{k}-1}$ to $x_{k}$ and from $x_{k}$ to $z_{k}$ respectively.

When the state-space is linear with Gaussian additive noise, the well-known Kalman filter achieves the solution for the optimal estimation problem. The Kalman filter is the most popular technique for handling linear models with Gaussian distributed noise. When the state-space can be written as a linear dynamic model with zero-mean Gaussian noise sources $\mathrm{v}_{\mathrm{k}}, \mathrm{N}\left(0, \mathrm{Q}_{\mathrm{k}}\right)$ and $\mathrm{w}_{\mathrm{k}}, \mathrm{N}\left(0, \mathrm{R}_{\mathrm{k}}\right)$, the posterior density is also Gaussian so it can be completely parameterized by its mean and covariance. Let $A_{k}$ and $B_{k}$ be two matrices defining a linear transformation for the process and observation equations. $Q_{k}$ and $R_{k}$ represent the process and observation noise covariance respectively.

The linear Gaussian state-space with a seasonal component can be written as:

$x_{k}=A_{k} x_{k-1}+\frac{2 \pi}{T} C_{k}+v_{k}$

$z_{k}=B_{K} x_{k}+w_{k}$

The Kalman filter computes the optimal conditional mean and covariance of $\mathrm{x}_{\mathrm{k}}$ by recursively predicting and updating a Gaussian belief distribution. The recursive method is optimal since using the following equations minimizes the mean square error of the observations and the predicted state.

The term $S_{k}$ denotes the covariance of an innovation matrix $\varepsilon_{\mathrm{k}}=\mathrm{z}_{\mathrm{k}}-\mathrm{B}_{\mathrm{k}} x_{\mathrm{k} \mid \mathrm{k}-1}$ that generates a sequence of uncorrelated terms. The superscript $T$ denotes matrix transposition and $\mathrm{K}_{\mathrm{k}}$ is the socalled Kalman gain. Both terms $\mathrm{S}_{\mathrm{k}}$ and $\mathrm{K}_{\mathrm{k}}$ can also be written as: 


$$
\begin{aligned}
& S_{k}=B_{k} \sum_{k \mid k-1} B_{k}^{T}+R_{k} \\
& K_{k}=\sum_{k \mid k-1} B_{k}^{T} S_{k}^{-1}
\end{aligned}
$$

\section{CONCLUSIONS}

Confidently identifying and determining values for discrete data points across a three dimensional plane to model climate variation is a non-trivial challenge for any single interpolation method. Outlying values that may not conform to the expected variations to a mean may in fact, be significant indicators of a change point yet to be observed. Kriging for instance, would prune such a value and complete the interpolation without including it in the cluster of predictors for new data point instances. Ensemble methods provide a multi algorithmic approach that does not discard any values until computations of all possible permutations of the data are exhausted. They also allow for a temporal variable to be meaningfully incorporated into the model without distorting the intrinsic geospatial properties of the former interpolation methods. Using a Kalman Filter to maintain data integrity and reduce noise in the data set during computation produces a clean and reliable model and a result. We hope to illustrate this by comparing the two model outputs when sufficient appropriate data is available from the WSN referred to here. Our work continues with installation of the WSN and continuing exploration of the interpolation methods and models using trivial data sets in order to gain insights to their dynamics.

\section{REFERENCES}

[1] Caprio, J M, and Quamme H A. (1998) Weather conditions associated with apple production in the Okanagan Valley of British Columbia., Agriculture and Agri-Food Canada Pacific Agri-Food Research Centre, Summerland, British Columbia, Canada, V0H 1Z0 1998, Vol. Contribution no.1075 129-137

[2] Van Leeuwen, C., Friant, P., Choné, X., Tregoat,O., Koundouras,S., and Dubourdieu,D. (2004) Influence of Climate, Soil, and Cultivar on Terroir Am. J. Enol. Vitic. 2004, pp.55:3:207

[3] Delyam, A.M. Chaotic Climate Dynamics. Lunivar Press, 2007. ISBN-13 978-1-905986-07-1

[4] Jones, G V, and Davis, R E,. (2000) Climate Influences on Grapevine Phenology, Grape Composition, and Wine Production and Quality for Bordeaux, France. Vols. Am. J. Enol. Vitic., Vol. 51, No. 3, 2000 pp249-261.

[5] Holton,J. An introduction to dynamic meteorology. Academic Press, 2004

[6] Berger, J. O., De Oliveira, V. and Sansó, B. (2001). Objective Bayesian analysis of spatially correlated data. Journal of the American Statistical Association, 96, 1361-1374

[7] Drignei, D. A kriging approach to the analysis of climate model experiments. (2009) Journal of Agricultural, Biological and Environmental Sciences. Springer New York 2009 Vol. 14(1) pp 99114. ISBN 1085-7117 (Print) 1537-2693 (online)

[8] Okun, Oleg; Valentini, Giorgio (Eds.) Supervised and Unsupervised ensemble methods and their applications IN Springer series, Studies in Computational Intelligence, vol 1262008

[9] Barry, RJ and Carleton, AM. "Synoptic and dynamic climatology" Routledge, 2001

[10] Silva, D, Meza, FJ and Varas E. Use of mesoscale model MM5 forecasts as proxies for surface meterorological and agroclimate variables. Cienc. Inv. Agr. [online], 2009, vol 36, no.3

[11] Grewal, M. S. Kalman Filtering: Theory \& Practice. Englewood Cliffs, NJ: Prentice-Hall, 1993

[12] Petrosyan, AS. GIS in meteorology and climatology. The needs and the challenges. European Geophysical Society. XXVI General Assembly. Nice, 25-30 March 2001

[13] Sallis, P., Jarur, M., and Trujillo, M.(2009). Frost prediction characteristics and classification using computational neural networks. In Australian Journal of Intelligent Information Processing Systems (AJIIPS) volume 10.1, 2008 (ISSN 1321-2133) pp50-58. Also published in M. Kppen et al. (Eds.): ICONIP 2008, Part I, LNCS 5506, 2009. Springer-Verlag Berlin Heidelberg 2009. pp. 1211-1220.

[14] National Institute of Water \& Atmospheric Research. The National Climate Database, National Institute of Water \& Atmospheric Research,. http://cliflo.niwa.co.nz/

[15] Ghobakhlou, A., Sallis, P., Diegel, O., Zandi, S. and Perera, A. (2009). Wireless sensor networks for environmental data monitoring. IEEE Sensor 2009 Conference 25-28 Oct 09, Christchurch, New Zealand. 